\title{
Perfil de risco imunológico de idosas com câncer de mama: os primeiros 37 casos
}

\author{
Immune risk profile of elderly women with breast cancer: the first 37 cases \\ Betina Vollbrecht ${ }^{1,2}$, Felipe Pereira Zerwes ${ }^{2,3,4}$, Janaína Ferreira Viegas ${ }^{2,3}$, Thiago Willers ${ }^{2,4}$, Ana Paula Ornaghi ${ }^{5}$, \\ Cristina Bonorino ${ }^{5}$, Antonio Luiz Frasson ${ }^{1,2,4}$
}

${ }^{1}$ Instituto de Geriatria e Gerontologia da Pontifícia Universidade Católica do Rio Grande do Sul (PUCRS), Porto Alegre, RS; ${ }^{2}$ Centro de Mama, Hospital São Lucas da PUCRS, Porto Alegre, RS; ${ }^{3}$ Instituto do Câncer do Sistema de Saúde Mãe de Deus, Porto Alegre, RS; ${ }^{4}$ Faculdade de Medicina da PUCRS, Porto Alegre, RS; ${ }^{5}$ Faculdade de Biociências e Instituto de Pesquisas Biomédicas, PUCRS, Porto Alegre, RS.

\section{RESUMO}

Objetivos: Avaliar o perfil imunológico de risco em idosas com câncer de mama e testar se este pode ser um fator preditivo confiável para determinar tipos de tratamento e seguimento oncológico.

Métodos: Foram pesquisadas a relação das células T CD4+/CD8+ e a sorologia para citomegalovírus no sangue periférico de mulheres com 60 anos ou mais de idade no momento do diagnóstico da neoplasia mamária, que realizaram tratamento cirúrgico no Centro de Mama da Pontifícia Universidade Católica do Rio Grande do Sul pelo Sistema Único de Saúde. Foram excluídas da pesquisa pacientes com sorologia positiva para HIV, com imunossupressão após transplante de órgãos e as que realizaram quimioterapia neoadjuvante. Os dados foram comparados em grupos conforme o comprometimento axilar, o tamanho tumoral, o perfil imunohistoquímico do tumor e a ocorrência de eventos adversos (recidiva axilar, recidiva local do tumor e/ou metástases). Nos casos de eventos adversos, foi realizada uma nova contagem de CD4+ e CD8+. Resultados: Foram incluídas 37 pacientes, entre as quais 10 tiveram metástases axilares. As pacientes com axila positiva para metástases apresentaram uma relação $\mathrm{CD} 4+/ \mathrm{CD} 8+$ maior que nos casos de axila negativa para metástases $(\mathrm{p}=0,04)$. Não foi encontrada diferença estatisticamente significativa em relação ao tamanho e perfil imunohistoquímico do tumor. No seguimento médio de 14,3 meses, ocorreram dois eventos adversos (uma recidiva axilar e um caso de metástases ósseas), quando se observou um aumento na relação das células T pesquisadas. Conclusões: A relação das células T CD4+/CD8+ parece aumentar nos casos de câncer de mama de pior prognóstico. Tanto quanto foi possível pesquisar na literatura, estes são os primeiros dados sobre células T CD4+ e CD8+ no sangue periférico de mulheres idosas com câncer de mama. Um seguimento maior poderá determinar o valor destas células como fator prognóstico e/ou preditivo.

DESCRITORES: LINFÓCITOS T CD4-POSITIVOS; LINFÓCITOS T CD8-POSITIVOS; RELAÇÃO CD4-CD8; CÂNCER DE MAMA/imunologia; GERIATRIA.

\section{ABSTRACT}

Aims: To evaluate the immune risk profile of elderly women with breast cancer and to assess whether this can be a reliable predictor to determine types of treatment and oncologic follow-up.

Methods: We assessed the CD4+/CD8+ ratio in peripheral blood cell, as well as serology for cytomegalovirus, of 37 women who were aged 60 years or more at the time they were diagnosed with breast cancer/. They all had surgical treatment at the Breast Center from Pontificia Universidade Catolica do Rio Grande do Sul. Those with positive serology for HIV, or immuno-suppressed due to organ transplant, as well as those who had neoadjuvant chemotherapy. Data was analyzed according to axillary involvement, tumor size, tumor immunohistochemical profile and occurrence of adverse events (axillary relapse, local relapse and/or metastases).

Results: The mean value of $\mathrm{CD} 4+/ \mathrm{CD} 8+$ ratio was 1.72 (min. 1.1, max. 2.32) and cytomegalovirus serology was positive in all subjects. Comparing the groups, patients with positive axillary metastases $(n=10)$ had a $\mathrm{CD} 4+/ \mathrm{CD} 8+$ ratio greater than in those with negative axillary metastases $(p=0.04)$. No statistically significant difference was detected regarding the size and immunohistochemical profile of the tumor. Two adverse events occurred at a mean follow-up of 14 months (one axillary relapse and one bone metastasis), when an increase in the CD4+/CD8+ ratio was observed.

Conclusions: The CD4+/CD8+ ratio appear to increase in cases of breast cancer with worst prognosis. As far as was possible to search, these are the first data on CD4+ and CD8+ peripheral blood of elderly women with breast cancer. A longer follow-up will determine the value of these cells as a prognostic and/or predictive marker.

KEY WORDS: CD4-POSITIVE T-LYMPHOCYTES; CD8-POSITIVE T-LYMPHOCYTES; CD4-CD8 RATIO; BREAST CANCER/immunology; GERIATRICS. 


\section{INTRODUÇÃO}

O sistema imune torna-se comprometido com a idade avançada, sendo menos eficaz no combate a neoplasias malignas. Isso resulta em maior índice de tumores malignos na população idosa, além de maior morbidade e mortalidade por causas oncológicas. ${ }^{1-4} \mathrm{~A}$ função das células T nos idosos está comprovadamente afetada, sendo uma característica do envelhecimento a diminuição no repertório desses linfócitos, com expansão de alguns clones com especificidade restrita. Uma taxa alterada de células T CD8+, aumentada em relação às $\mathrm{CD} 4+$ (normalmente mais frequentes no sangue) vem sendo associada com risco imunológico no idoso, e evidências recentes sugerem que as infecções virais por vírus da família do citomegalovírus (CMV), recorrentes ao longo da vida, podem ser determinantes nestas alterações. ${ }^{5,6}$

Nesse intenso processo de envelhecimento populacional, sabemos que a população idosa é predominantemente feminina. Com o avançar da idade aumentam as chances de determinadas doenças, como as patologias cardíacas, respiratórias e alguns tipos de câncer. Especificamente na população feminina idosa, a neoplasia de maior incidência é o câncer de mama, cuja incidência aumenta proporcionalmente com a idade. ${ }^{1-4}$ A chance de diagnosticarmos um câncer de mama em uma mulher de 30 anos de idade é de um caso em 2212 mulheres; já com 60 anos é de um caso a cada 23 mulheres, e com mais de 80 anos de um caso a cada 8 mulheres. ${ }^{1}$ Alterações no sistema imune podem ser responsáveis por este aumento de neoplasia mamária nas mulheres com mais de 60 anos de idade..$^{1,5}$

$\mathrm{O}$ conceito de perfil imunológico de risco tem sido considerado quando há uma relação de células $T$ CD4+/CD8+ menor que 1 , associado com sorologia positiva para CMV. A literatura descreve a prevalência de risco imunológico entre 15 e $20 \%$ da população em geral. ${ }^{5,16-18,21}$ Tanto quanto foi possível pesquisar, até este momento não há dados na literatura especificamente sobre a taxa $\mathrm{CD} 4 / \mathrm{CD} 8$ no sangue periférico de pessoas com neoplasia maligna. Especificamente, não há dados na literatura, até a presente data, sobre a análise da relação de células $\mathrm{T}$ CD4+/CD8+ e a sorologia para CMV em mulheres idosas com neoplasia mamária.

Neste estudo propomos analisar o perfil de risco imunológico de mulheres idosas e testar se o mesmo pode ser um fator preditivo confiável para determinar tipos de tratamento e seguimento oncológico. Os resultados obtidos podem fornecer elementos para elaboração de novas políticas de saúde de atenção às idosas com câncer de mama. Assim, o objetivo principal do estudo foi estudar o perfil imunológico das mulheres idosas com câncer de mama, determinando a relação das células T CD4+/CD8+ e a sorologia para CMV. Como objetivos secundários, este estudo investigou a associação entre o perfil imunológico, o comprometimento linfonodal, o tamanho tumoral e o perfil imunohistoquímico. Avaliando se existe um perfil imunológico de maior risco para recidiva local e metástases, o estudo procurou determinar se a dosagem de células T CD4+ e CD8+ pode ser um fator preditivo e/ou prognóstico para neoplasia maligna de mama.

\section{MÉTODOS}

Este foi um estudo piloto, que incluiu mulheres com diagnóstico de câncer de mama que realizaram tratamento cirúrgico no Centro de Mama da Pontifícia Universidade Católica do Rio Grande do Sul (CEMAPUCRS) pelo Sistema Único de Saúde (SUS) com 60 anos ou mais de idade no momento do diagnóstico. Foram excluídas da pesquisa pacientes com sorologia positiva para HIV, com imunossupressão após transplante de órgãos e as que realizaram quimioterapia neoadjuvante.

A pesquisa foi iniciada após aprovação do Comitê de Ética em Pesquisa da PUCRS (parecer consubstanciado de número 154.305). As pacientes assinaram um Termo de Consentimento Livre e Esclarecido, através do qual receberam o conhecimento dos objetivos do estudo, riscos e seguimento. As pacientes que concordaram em participar do estudo realizam o seguimento de rotina no CEMA-PUCRS para detectar eventos adversos: recidiva local do câncer de mama, recidivas axilares, metástases e óbito.

Foram coletadas amostras de $10 \mathrm{ml}$ de sangue venoso no pré-operatório. $\mathrm{O}$ sangue foi processado para isolamento de células mononucleares e isolamento de plasma no Laboratório de Imunologia Celular e Molecular da PUCRS. Todas as pacientes tiveram suas células marcadas com anticorpos CD4+/CD8+ e sorologia para CMV. Nos casos que apresentaram recidiva local, axilar e lesões metastáticas foram coletadas novas amostras de sangue.

Os dados foram tabulados e analisados através de computador utilizando o programa Microsoft Office Excel e o programa Epi Info. Para as variáveis quantitativas foram calculadas médias ou medianas e medidas de dispersão. Os dados qualitativos foram sumariados através de frequência em cada classe. Os testes estatísticos utilizados foram o teste " $t$ " de Student para amostras pareadas e independentes e variáveis quantitativas; teste não paramétrico de Mann Whitney 
para variáveis quantitativas que não satisfizeram as exigências do teste $\mathrm{t}$; teste de Wilcoxon-Mann-Whitney para amostras independentes; teste do qui-quadrado, para variáveis categóricas; teste "Exato de Fisher", para categóricas dicotômicas que não satisfizeram as exigências do qui-quadrado. Foram considerados significativos valores $\mathrm{p}$ menores que 0,05 .

\section{RESULTADOS}

Conforme os critérios de inclusão, foram selecionadas para participar da pesquisa 37 mulheres, com idade média de 64 anos e mediana de 67 anos (intervalo interquartil 62 a 83 anos) no momento do diagnóstico do câncer de mama. $\mathrm{O}$ tratamento cirúrgico da mama foi ressecção segmentar em 19 (51,4\%) pacientes, mastectomia em 15 (40,5\%) e adenomastectomia em três $(8,1 \%)$ pacientes. A biópsia do linfonodo sentinela (BLS) foi realizada em 36 pacientes, entre as quais em nove $(25 \%)$ o linfonodo sentinela foi positivo para metástases, sendo que uma paciente foi submetida diretamente ao esvaziamento axilar completo por apresentar uma axila clinicamente positiva no momento da cirurgia (Tabela 1). O esvaziamento axilar completo foi realizado em seis $(16,7 \%)$ pacientes. As três $(8,3 \%)$ pacientes com linfondo sentinela positivo que não realizaram esvaziamento axilar apresentavam critérios de tratamento conforme o estudo ACOSOG Z0011. ${ }^{23}$ O tamanho tumoral médio foi de 2,24 $(0,2-8,5) \mathrm{cm}$, sendo em $16(43,2 \%)$ casos foi menor que $2 \mathrm{~cm}$. Os diagnósticos anatomopatológicos estão descritos na Tabela 1.

O perfil imunohistoquímico do tumor foi classificado conforme os receptores hormonais $(\mathrm{RH})$, a expressão do oncogene Her-2/neu e do Ki67. Foram calculadas as frequências de cada perfil e a correspondência do mesmo com a relação CD4+/CD8+ (Tabela 2).

Como tratamento adjuvante, todas as pacientes que realizaram cirurgia conservadora de mama e adenomastectomia receberam radioterapia; das pacientes mastectomizadas $(\mathrm{n}=15)$, a radioterapia complementar foi realizada em oito casos. Todas as mulheres com $\mathrm{RH}$ positivo $(\mathrm{n}=28 ; 75 \%)$ receberam hormonioterapia, todas Her-2/neu positivo $(n=9$; $24,3 \%$ ) receberam trastuzumab. E a quimioterapia adjuvante foi aplicada em $23(62,1 \%)$ pacientes.

No seguimento médio de 14 meses ocorreu uma $(2,7 \%)$ recidiva axilar e uma $(2,7 \%)$ paciente foi diagnosticada com metástases ósseas. Não ocorreram recidivas locais. A relação dos linfócitos $\mathrm{T}$ CD4+/ CD8+ média foi de 1,72 (mínimo 1,1-máximo 2,3).
Todas as pacientes (100\%) apresentaram IgG reagente para CMV. A comparação das médias da relação CD4+/CD8+ conforme a positividade para metástases, o tamanho do tumor e o perfil imunohistoquímico do tumor estão descritos na Tabela 2.

Ocorreram dois eventos adversos no decorrer do estudo. A paciente com recidiva axilar apresentava a relação de células T CD4+/CD8+ de 1,2 no momento do diagnóstico e 1,7 na recidiva axilar e sorologia positiva para CMV. Essa paciente tinha idade de 61 anos, recidivou em 14 meses, foi tratada com esvaziamento axilar radical e está viva sem evidência de doença há seis meses. A paciente diagnosticada com metástases ósseas tinha 68 anos, apresentou no momento do diagnóstico do tumor mamário a relação CD4+/CD8+ de 1,9 e, quando evidenciadas as metástases ósseas, esta relação foi de 2,2. No tempo de seguimento médio de 14 meses deste estudo, todas as pacientes incluídas estavam vivas e sem outros eventos adversos.

Tabela 1. Tratamento cirúrgico e características clínicopatológicas do câncer de mama em 37 pacientes idosas.

\begin{tabular}{lc}
\multicolumn{1}{c}{ Características } & $\mathrm{n}(\%)$ \\
\hline $\begin{array}{l}\text { Tratamento cirúrgico da mama } \\
\text { Ressecção segmentar }\end{array}$ & $19(51,4)$ \\
Mastectomia & $15(40,5)$ \\
Adenomastectomia & $3(8,1)$ \\
Metástases axilares & \\
Ausente & $27(73,0)$ \\
Presente & $10(27,0)$ \\
Diagnóstico anatomopatológico & \\
Carcinoma ductal invasor & $32(86,5)$ \\
Carcinoma lobular invasor & $3(8,1)$ \\
Carcinoma medular & $1(2,7)$ \\
Carcinoma tubular & $1(2,7)$ \\
\hline
\end{tabular}

Tabela 2. Média da relação de células T CD4+/CD8+ em 37 pacientes idosas com câncer de mama, conforme presença ou ausência de metástases axilares, tamanho tumoral e perfil imunohistoquímico do tumor.

\begin{tabular}{lccc} 
Características do tumor & $\mathrm{n}$ & $\begin{array}{c}\text { Relação } \\
\text { CD4+/CD8 }+\end{array}$ & $p^{*}$ \\
\hline $\begin{array}{l}\text { Presença de metástases axilares } \\
\quad \text { Axila negativa para metástases }\end{array}$ & 27 & 1,58 & $0,04^{*}$ \\
$\quad$ Axila positiva para metástases & 10 & 1,98 & \\
Tamanho do tumor & & & \\
$\quad$ Tumor $<2 \mathrm{~cm}$ & 16 & 1,60 & $0,26^{*}$ \\
$\quad$ Tumor $>2 \mathrm{~cm}$ & 21 & 1,79 & \\
Perfil imunohistoquímico do tumor & & & \\
$\quad$ Luminal A & 14 & 1,51 & \\
$\quad$ Luminal B & 10 & 1,71 & $0,22^{*}$ \\
$\quad$ Her-2/neu positivo & 9 & 1,89 & \\
$\quad$ Triplo negativo & 4 & 1,62 & \\
\hline
\end{tabular}

* Teste t de Student. 


\section{DISCUSSÃO}

O grupo de pacientes incluídas neste estudo, por serem mulheres idosas, apresentam características tumorais de bom prognóstico. O tipo histológico mais comum foi CDI, RH positivo e Her-2/neu negativo, exatamente como esperado nesta faixa etária. ${ }^{1,26,27}$ Para esse grupo apresentar eventos adversos como recidiva local, recidivas axilares e/ou metástases é necessário um seguimento mais longo. O seguimento, até a conclusão do presente estudo, foi curto (média de 14 meses), o que pode influir na baixa ocorrência de eventos adversos. Assim, o objetivo de verificar se o perfil imunológico das pacientes pode ser usado como fator preditivo e/ou prognóstico em câncer de mama ficou prejudicado pelo curto espaço de tempo observado.

Merece destaque nesse grupo de pacientes o tamanho tumoral. Na maioria das pacientes, o tumor apresentou tamanho maior que $2 \mathrm{~cm}$. Isto reflete um diagnóstico de lesões mais avançadas, uma falha nos programas de rastreamento. Infelizmente, verifica-se falha no diagnóstico precoce de câncer de mama no sistema de saúde pública do Brasil. Por serem pacientes idosas, o crescimento tumoral costuma ser lento, logo parece haver realmente um retardo no diagnóstico clínico e/ou por exame de imagem.

A relação dos linfócitos $\mathrm{T}$ CD4+/CD8+ mínima foi de 1,1 portanto, nenhuma paciente apresentou por conceito perfil imunológico de risco (relação CD4+/CD8+ menor que 1). ${ }^{15,21,28} \mathrm{Em}$ todos os grupos comparados nesta pesquisa, a relação $\mathrm{CD} 4+/ \mathrm{CD} 8+$ foi maior nas pacientes com pior prognóstico: tamanho tumoral maior que $2 \mathrm{~cm}$, axila positiva, tumores Her-2/neu positivo. A diferença foi estatisticamente significativa entre os grupos axila negativa e positiva para metástases $(\mathrm{p}=0,04)$. Inclusive nos dois eventos adversos, esta relação aumentou no momento do diagnóstico da recidiva axilar e no diagnóstico da metástase óssea. Estes dados parecem demonstrar um aumento da atividade do sistema imunológico nas pacientes de pior prognóstico. Tanto quanto foi possível pesquisar, até este momento não há dados na literatura especificamente sobre a taxa CD4+/CD8+ no sangue periférico de mulheres com diagnóstico de câncer de mama. Assim, não conseguimos comparar nossos dados com outras pesquisas.

Existem estudos sobre as células T-reg no sangue periférico de pacientes com diversas neoplasias malignas. Em uma pesquisa conduzida por Decker et al., ${ }^{30}$ foi analisado o número de células T-reg no sangue periférico de 292 pacientes recentemente diagnosticadas com câncer da mama. Os números de T-reg no sangue periférico de pacientes Her-2/neu positivo foram mais elevados do que em amostras de pacientes com $\mathrm{RH}$ positivo e Her-2/neu negativo..$^{30} \mathrm{Os}$ mecanismos que levam à expansão das células T-regs em pacientes com Her-2/neu positivo não foi definido na literatura até este momento. No entanto, alguns estudos já demonstraram que as células de câncer de mama Her-2/neu positivo são especialmente capazes de estimular respostas imunológicas. ${ }^{28-30}$

Em conclusão, neste estudo piloto sobre o perfil imunológico de pacientes idosas com câncer de mama, houve um aumento na relação $\mathrm{CD} 4+/ \mathrm{CD} 8+$ nas pacientes com axila positiva para metástases. Para determinar se a relação dessas células pode ser usada como um fator preditivo e/ou prognóstico para câncer de mama, estamos recrutando mais pacientes e realizando um seguimento mais longo. Por ser um trabalho pioneiro na literatura, acreditamos ser importante a publicação destes dados iniciais com o objetivo de estimular outros centros de mastologia e imunologia a pesquisarem também células T CD4+e CD8+ em associação ao câncer de mama.

\section{REFERÊNCIAS}

1. Crivellari D, Aopro M, Leonard R, Minckwitz G, Brain E, Goldhirsch A, Veronesi A, Muss H. Breast Cancer in the eldery. Jour Clin Oncology 2007;25(14):1882-90.

2. Kaufmann M, Morrow M, Mickwitz G, Harris JR. Locoregional Treatment of Primary Breast Cancer. Cancer 2010;116(5):1184-91.

3. Hansen D, Viegas J, Vollbrecht B, Frasson A. Câncer de mama em mulheres idosas. In: Schwanke CHA, Gomes I, Pedro REL, organizadores. Atualizações em Geriatria e Gerontologia II: abordagens multidisciplinares e interdisciplinares. Porto Alegre: Edipucrs; 2009. p. 47-63.

4. Abdo C. Estudo Populacional do envelhecimento no Brasil. São Paulo: Segmento Farma; 2009.

5. Saltzman RL, Peterson PK. Immunodeficiency of the elderly. Rev Infect Dis 1987;9(6):1127-39.

6. Schwanke CHA, Gomes I, Pedro REL, organizadores. Atualizações em Geriatria e Gerontologia II: abordagens multidisciplinares e interdisciplinares. Porto Alegre: Edipucrs; 2009.

7. HayFlick L. Como e por que envelhecemos. Rio de Janeiro: Campos; 1997.

8. Bynoe MS, Bonorino P, Viret C. Control of experimental autoimmune encephalomyelitis by CD4+ suppressor T cells: peripheral versus in situ immunoregulation. J Neuroimmunol 2007;191(1-2):61-9. 
9. Paula C, Motta A, Schmitz C, Nunes CP, Souza AP, Bonorino C. Alterations in dendritic cell function in aged mice: potential implications for immunotherapy design. Biogerontology 2009;10(1):13-25.

10. Khan N, Shariff N, Cobbold M, Bruton R, Ainsworth JA, Sinclair AJ, et al. Cytomegalovirus seropositivity drives the CD8 T cell repertoire toward greater clonality in healthy elderly individuals. J Immunol 2002;169(4):1984-92.

11. Callahan JE, Kappler JW, Marrack P. Unexpected expansions of CD8-bearing cells in old mice. J Immunol 1993;151(12):6657-69.

12. Clambey ET, van Dyk LF, Kappler JW, Marrack P. Non-malignant clonal expansions of CD8+ memory T cells in aged individuals. Immunol Rev 2005;205:170-89.

13. Clambey ET, Kappler JW, Marrack P. CD8 T cell clonal expansions \& aging: a heterogeneous phenomenon with a common outcome. Exp Gerontol 2007;42(5):407-11.

14. Burkett PR, Koka R, Chien M, Chai S, Boone DL, Ma A. Coordinate expression and trans presentation of interleukin (IL)-15Ralpha and IL-15 supports natural killer cell and memory CD8+ T cell homeostasis. J Exp Med 2004;200(7):825-34.

15. Pawelec G, Akbar A, Caruso C, Solana R, Grubeck-Loebenstein B, Wikby A. Human immunosenescence: is it infectious? Immunol Rev 2005;205:257-68.

16. Rufer N, Zippelius A, Batard P, Pittet MJ, Kurth I, Corthesy P, Cerottini JC, Leyvraz S, Roosnek E, Nabholz M, Romero P. Ex vivo characterization of human CD8+ T subsets with distinct replicative history and partial effector functions. Blood 2003;102(5):1779-87.

17. Koch SD, Kempf J, Pawelec G. T Cell Immunity and Aging. Sci Aging Knowledge Environ 2005;2005(48):pe37.

18. Fulop T, Larbi A, Wikby A, Mocchegiani E, Hirokawa K, Pawelec G. Dysregulation of T-cell function in the elderly: scientific basis and clinical implications. Drugs Aging 2005;22(7):589-603.

19. Ouyang Q, Wagner WM, Wikby A, Walter S, Aubert G, Dodi AI, Travers P, Pawelec G. Large numbers of dysfunctional CD8+ T lymphocytes bearing receptors for a single dominant CMV epitope in the very old. J Clin Immunol 2003; 23(4):247-57.

20. Derhovanessian E, Theeten H, Hahnel K, Van Damme P, Cools N, Pawelec G. Cytomegalovirus associated accumitation of late differentiated CD4 T cells correlates with poor humoral response to influenza vaccinations. Vaccine 2013;31(4):685-90.

21. Pawelec G, Barnett Y, Forsey R, Frasca D, Globerson A, McLeod J, Caruso C, Franceschi C, Fülöp T, Gupta S, Mariani E, Mocchegiani E, Solana R. T cells and aging, January 2002 update. Front Biosci 2002; 7:d1056-183.

22. Utrera-Barillas D, Valdez-Salazar HA, Gómez-Rangel D, Alvarado-Cabrero I, Aguilera P, Gómez-Delgado A, Ruiz-Tachiquin ME. Is human cytolegalovirus associated with breast cancer progression? Infect Agent Cancer. 2013;8(1):12.

23. Giuliano AE, Hunt KK, Ballman KV, Beitsch PD, Whitworth PW, Blumencranz PW, Leitch AM, Saha S, McCall LM, Morrow M. Axillarry Dissections ve no axillary dissection in women with invasive breast cancer and sentinela node mateastases - a randomized clinical trial. JAMA 2011;305(6):569-75.

24. Sauter G, Lee J, Bartlett JM, Slamon DJ, Press MF. Guidelines for human epidermal growth factor receptor 2 testing: biologic and methodologic considerations. J Clin Oncol 2009;27:1323-33.

25. Hammond ME, Hayes DF, Dowsett M, Allred DC, Hagerty KL, Badve S, Fitzgibbons PL, Francis G, Goldstein NS, Hayes M, Hicks DG, Lester S, Love R, Mangu PB, McShane L, Miller K, Osborne CK, Paik S, Perlmutter J, Rhodes A, Sasano H, Schwartz JN, Sweep FC, Taube S, Torlakovic EE, Valenstein P, Viale G, Visscher D, Wheeler T, Williams RB, Wittliff JL, Wolff AC. American Society of Clinical Oncology/ College Of American Pathologists guideline recommendations for immunohistochemical testing of estrogen and progesterone receptors in breast cancer. J Clin Oncol. 2010;28(16):2784-95.

26. Wildiers H, Kunkler I, Biganzoli L, Fracheboud J, Vlastos G, Bernard-Marty C, Hurria A, Extermann M, Girre V, Brain E, Audisio RA, Bartelink H, Barton M, Giordano SH, Muss H, Aapro M; International Society of Geriatric Oncology. Management of breast cancer in elderly individuals: recommendations of the International Society of Geriatric Oncology. Lancet Oncol. 2007;8(12):1101-15.

27. Lima LNB, Vollbrecht B, Machado LS, Millen E, Frasson A. Câncer de mama em mulheres idosas. In: Frasson A, editor. Doenças da mama: guia de bolso baseado em evidências. São Paulo: Atheneu; 2013. p. 379-82.

28. Pawelec G. Hallmarks of human "immunosenescence”: adaptation or dysregulation? Immun Ageing 2012;9(1):15.

29. Sellitto A, Galizia G, De Fanis U, Lieto E, Zamboli A, Orditura M, De Vita F, Giunta R, Lucivero G, Romano C. Behavior of circulating CD4+ CD25+ Foxp3+ regulatory T cells in colon cancer patients undergoing surgery. J Clin Immunol 2012;31:1095-104.

30. Decker T, Fischer G, Bucke W, Bucke P, Stotz F, Gruneberger A, Gropp-Meier M, Wiedemann G, Pfeiffer C, Peschel C, Gotze K. Increased number of regulatory $\mathrm{T}$ cells (T-regs) in the peripheral blood of patients with Her-2/neu-positive early breast cancer. J Cancer Res Clin Oncol 2012;138(11):1945-50. 